\title{
ON THE LOWER BOUND OF THE NUMBER OF REAL ROOTS OF A RANDOM ALGEBRAIC EQUATION WITH INFINITE VARIANCE. II
}

G. SAMAL AND M. N. MISHRA

ABSTRACT. Let $N_{n}$ be the number of real roots of a random algebraic equation $\sum_{v=0}^{n} \xi_{v} x^{v}=0$ where the $\xi_{v}$ 's are independent random variables with a common characteristic function

$$
\exp \left(-C|t|^{\alpha}\right), \quad \alpha>1,
$$

and $C$, a positive constant. Then for $n>n_{0}$,

$$
N_{n}>(\mu \log n) /(\log \log n)
$$

outside a set of measure at most

$$
\mu^{\prime} /\left\{\log \left(\left(\log n_{0}\right) /\left(\log \log n_{0}\right)\right)\right\}^{x-1} .
$$

1.1. Introduction. Let $N_{n}$ be the number of real roots of a random algebraic equation $\Sigma_{0}^{n} \xi_{v} x^{v}=0$, where the coefficients $\xi_{v}$ 's are independent random variables with identical distributions.

Taking the coefficients as normally distributed or uniformly distributed in $[-1,1]$ or assuming the values +1 and -1 with equal probabilities, Littlewood and Offord [3] have shown that $N_{n}>(\alpha \log n) /(\log \log \log n)$, except for a set of measure at most $A / \log n, n$ being sufficiently large.

Taking the coefficients as normally distributed, Evans [1] has shown that there exists an integer $n_{0}$ such that for $n>n_{0}$,

$$
N_{n}>(\beta \log n) /(\log \log n)
$$

except for a set of measure at most $\left(B \log \log n_{0}\right) /\left(\log n_{0}\right)$.

Samal [4] has considered the general case when the $\xi_{v}$ 's have expectation zero with variance and third absolute moment nonzero finite. He has shown that $N_{n}>\varepsilon_{n} \log n$ where $\varepsilon_{n} \rightarrow 0$ but $\varepsilon_{n} \log n \rightarrow \infty$ as $n$ tends to infinity. The measure of the exceptional set tends to zero as $n$ tends to infinity.

Recently, Samal and Mishra [5] have considered the case when the $\xi_{v}$ 's have a common characteristic function $\exp \left(-C|t|^{\alpha}\right)$ where $C$ is a positive constant and $\alpha \geqq 1$. They have obtained that

$$
N_{n}>(\mu \log n) /(\log \log n) \text {. }
$$

Received by the editors October 13, 1971.

AMS 1969 subject classifications. Primary:60xx, 6090.

Key words and phrases. Random equation, infinite variance, real zeros, lower bound. 
Simple calculation will show that their exceptional set is of measure at most $\mu^{\prime} /(\log \log n)(\log n)^{\alpha-1}$ if $1 \leqq \alpha<2$ and $\left(\mu^{\prime} \log \log n\right) /(\log n)$ if $\alpha \geqq 2$, $n$ being sufficiently large.

Our object is to show in this case that for $n>n_{0}$,

$$
N_{n}>(\mu \log n) /(\log \log n)
$$

except for a set of measure at most $\mu^{\prime} /\left\{\log \left(\left(\log n_{0}\right) /\left(\log \log n_{0}\right)\right)\right\}^{\alpha-1}$, when $\alpha>1$.

Our result is true for all $\alpha>1$, but its importance lies in the range $1<\alpha<2$ when the variance is infinite. However, incidentally we find that the corresponding result of Evans [1] happens to be a special case of our result when $\alpha=2$, although our exceptional set is larger than his.

Positive constants shall be denoted by $\mu$ 's. We shall suppose that any inequality is satisfied when $n$ is large.

Our proofs are modifications of our earlier ones. At some places, we shall indicate only the conclusion arrived at and try to avoid repetition of similar arguments given in the paper cited above.

2.1. THEOREM. Let $f(x)$ be a polynomial of degree $n$ whose coefficients are independent random variables with a common characteristic function $\exp \left(-C|t|^{\alpha}\right)$, where $\alpha>1$ and $C$ is a positive constant. Then there exists an integer $n_{0}$ such that for each $n>n_{0}$, the number of real roots of the equation $f(x)=0$ is at least $(\mu \log n) /(\log \log n)$ except for a set of measure at most $\mu^{\prime} /\left\{\log \left(\left(\log n_{0}\right) /\left(\log \log n_{0}\right)\right)\right\}^{\alpha-1}$.

LEMMA 2.1. If a random variable $\xi(u)$ has characteristic function $\exp \left(-C|t|^{\alpha}\right)$ then

$$
\operatorname{Pr}(|\xi(u)|>\varepsilon)<\frac{2^{1+\alpha} C}{1+\alpha} \cdot \frac{1}{\varepsilon^{\alpha}}
$$

for every $\varepsilon>0$.

For proof see Samal and Mishra [5].

2.2. Proof of THE Theorem. Take constants $A$ and $B$ such that $0<B<1$ and $A>1$. Let

$$
\lambda_{m}=m^{1 / x} \log m
$$

Let $M_{m}, m=1,2,3, \cdots$, be a sequence of integers defined by

$$
M_{m}=\left[\left(2^{\alpha} A e \lambda_{m}^{\alpha}\right) /(B m)\right]+1
$$

and $k$ be the integer defined by

$$
(2 k) ! M_{n}^{2 k} \leqq n<(2 k+2) ! M_{n}^{2 k+2} .
$$


It follows from this that

$$
\left(\mu_{1}^{\prime} \log n\right) /(\log \log n)<k<\left(\mu_{2}^{\prime} \log n\right) /(\log \log n) .
$$

Hence $k$ is large when $n$ is large.

We consider $f=\sum_{v=0}^{n} \xi_{v} x^{v}$ at the points

$$
x_{m}=\left(1-1 /\left((2 m) ! M_{m}^{2 m}\right)\right)^{1 / \alpha}, \text { for } m=\left[\frac{1}{2} k\right]+1,\left[\frac{1}{2} k\right]+2, \cdots, k .
$$

We have

$$
f\left(x_{m}\right)=U_{m}+R_{m}
$$

where

$$
U_{m}=\sum_{(2 m-1) ! M_{m}{ }^{2 m-1}+1}^{(2 m+1) ! M_{m}{ }^{2 m+1}} \xi_{v} x_{m}^{v}
$$

and

Let

$$
R_{m}=\left(\sum_{0}^{(2 m-1) ! M_{m}^{2 m-1}}+\sum_{(2 m+1) ! M_{m}{ }^{2 m+1}+1}^{n}\right) \xi_{v} x_{m}^{v} .
$$

$$
V_{m}=\left(\sum_{(2 m-1) ! M_{m}{ }^{2 m-1}+1}^{(2 m+1) ! M M_{m}^{2 m+1}} \xi_{v} x_{m}^{\alpha v}\right)^{1 / \alpha} .
$$

2.3. The Lemma 2 and Lemma 3 of Samal and Mishra [5] shall be modified in the following manner.

LEMMA 2.2 .

$$
\left|\sum_{(2 m+1) !: M_{m}^{2 m+1}+1}^{n} \xi_{v} x_{m}^{v}\right|<\frac{1}{2} V_{m}
$$

except for a set of measure at most

$$
\left(2^{1+2 \alpha} \cdot C /(1+\alpha)\right)(A e / B) e^{-(2 m-1) M_{m}}
$$

for every sufficiently large $m$.

LEMMA 2.3 .

$$
\left|\sum_{0}^{(2 m-1) ! M M_{m}{ }^{2 m-1}} \xi_{v} x_{m}^{v}\right|<\lambda_{m}\left(\sum_{0}^{(2 m-1) ! M M_{m}^{2 m-1}} x_{m}^{\alpha v}\right)^{1 / \alpha}
$$

except for a set of measure at most $2^{1+\alpha} \cdot C /(1+\alpha) \lambda_{m}^{\alpha}$.

Proofs of these two lemmas will run in the same line as in the paper cited above. 
2.4. Thus for every sufficiently large $m$,

$$
\left|R_{m}\right|<\frac{1}{2} V_{m}+\lambda_{m}\left(\sum_{0}^{(2 m-1) ! M_{m}^{2 m-1}} x_{m}^{\alpha v}\right)^{1 / \alpha}
$$

outside a set of measure at most $\mu_{1} e^{-(2 m-1) \cdot l_{m}}+\mu_{2} / \hat{\lambda}_{m}^{\alpha}$. But

$$
\begin{aligned}
\lambda_{m}\left(\sum_{0}^{(2 m-1) ! M_{m}{ }^{2 m-1}} x_{m}^{\alpha x}\right)^{1 / \alpha} & <\lambda_{m}\left\{(2 m-1) ! M_{m}^{2 m-1}+1\right\}^{1 / \alpha} \\
& \leqq \lambda_{m} \cdot 2^{1 / \alpha}\left\{(2 m-1) ! M_{m}^{2 m-1}\right\}^{1 / \alpha} \\
& \leqq \lambda_{m} \cdot 2^{1 / \alpha}\left\{\frac{(A e / B) V_{m}^{\alpha}}{2 m M_{m}}\right\}^{1 / \alpha}<\frac{1}{2} V_{m} .
\end{aligned}
$$

The last step follows from (2.2) and from the relation

$$
V_{m}^{x} \geqq(B / A e)(2 m) ! M_{m}^{2 m} .
$$

Since $k \rightarrow \infty$ as $n \rightarrow \infty$, it follows that when $n$ is sufficiently large $\left|R_{m}\right|<V_{m}$ for $m=\left[\frac{1}{2} k\right]+1,\left[\frac{1}{2} k\right]+2, \cdots, k$ except for a set of measure at most $\mu_{1} e^{-(2 m-1) \cdot I_{m}}+\mu_{2} / \lambda_{m}^{\alpha}$

We define $E_{m}$ and $F_{m}$ as follows:

$$
\begin{aligned}
& E_{m}=\left\{U_{2 m}>V_{2 m}, U_{2 m+1}<-V_{2 m+1}\right\}, \\
& F_{m}=\left\{U_{2 m}<-V_{2 m}, U_{2 m+1}>V_{2 m+1}\right\} .
\end{aligned}
$$

It can be shown as in Samal and Mishra [5] that $m\left(E_{m} \cup F_{m}\right)=\delta>0$, where $\delta$ is independent of $m$.

Let $\eta_{m}$ be a random variable which takes value 1 on $E_{m} \cup F_{m}$ and zero elsewhere. In other words,

$$
\begin{aligned}
\eta_{m}=1 & \text { with probability } \delta, \\
& =0 \quad \text { with probability } 1-\delta .
\end{aligned}
$$

Let $\zeta_{m}$ be defined as follows

$$
\begin{aligned}
& \zeta_{m}=0 \text { if }\left|R_{2 m}\right|<V_{2 m} \text { and }\left|R_{2 m+1}\right|<V_{2 m+1}, \\
& =1 \text { otherwise. }
\end{aligned}
$$

Let $\xi_{m}=\eta_{m}-\eta_{m} \zeta_{m}$. If $\xi_{m}=1$, there is a root of the polynomial in the interval $\left(x_{2 m}, x_{2 m+1}\right)$. Hence the number of roots in the interval $\left(x_{2 m_{0}}, x_{2 k+1}\right)$ where $m_{0}=\left[\frac{1}{2} k\right]+1$ must exceed $\sum_{m=m_{0}}^{k} \xi_{m}$.

2.5. We shall need the strong law of large numbers in the following form.

Let $\eta_{1}, \eta_{2}, \cdots$ be a sequence of independent random variables identically 
distributed with $V\left(\eta_{i}\right)<1$ for all $i$, then for each $\varepsilon>0$,

$$
\operatorname{Pr}\left\{\sup _{k \geqq k_{0}}\left|\frac{1}{k} \sum_{i=1}^{k}\left\{\eta_{i}-E\left(\eta_{i}\right)\right\}\right| \geqq \varepsilon\right\} \leqq \frac{D}{\varepsilon^{2} k_{0}},
$$

where $D$ is a positive constant.

Here we have

$$
\begin{aligned}
\sum_{m=m_{0}}^{k}\left\{\xi_{m}-E\left(\eta_{m}\right)\right\} & =\sum_{m=m_{0}}^{k}\left\{\eta_{m}-\eta_{m} \zeta_{m}-E\left(\eta_{m}\right)\right\} \\
& =\sum_{m=m_{0}}^{k}\left\{\eta_{m}-E\left(\eta_{m}\right)\right\}-\sum_{m=m_{0}}^{k} \eta_{m} \zeta_{m} .
\end{aligned}
$$

Therefore,

$$
\begin{aligned}
\left|\sum_{m=m_{0}}^{k}\left\{\xi_{m}-E\left(\eta_{m}\right)\right\}\right| & \leqq\left|\sum_{m=m_{0}}^{k}\left\{\eta_{m}-E\left(\eta_{m}\right)\right\}\right|+\left|\sum_{m=m_{0}}^{k} \eta_{m} \zeta_{m}\right| \\
& \leqq\left|\sum_{m=m_{0}}^{k}\left\{\eta_{m}-E\left(\eta_{m}\right)\right\}\right|+\sum_{m=m_{0}}^{k} \zeta_{m} .
\end{aligned}
$$

Since

$$
\begin{aligned}
E\left(\zeta_{m}\right) & =1 \cdot \operatorname{Pr}\left(\zeta_{m}=1\right) \leqq \operatorname{Pr}\left(\left|R_{m}\right| \geqq V_{m}\right) \\
& \leqq \mu_{1} e^{-(2 m-1) I_{m}}+\mu_{2} / \lambda_{m}^{\alpha}
\end{aligned}
$$

we have $\sum_{m=m_{0}}^{k} \zeta_{m}<\left(k-m_{0}+1\right) \varepsilon_{1}$, outside an exceptional set of measure at most

$$
\begin{aligned}
& \sum_{m=m_{0}}^{k} \frac{1}{\left(l-m_{0}+1\right) \varepsilon_{1}}\left(\mu_{1} e^{-(2 m-1) . I_{m}}+\frac{\mu_{2}}{\lambda_{m}^{\alpha}}\right) \\
&<\frac{1}{\varepsilon_{1}}\left\{\mu_{1} e^{-\left(2 m_{0}-1\right) M_{m_{0}}}+\mu_{2} / x_{m_{0}}^{\alpha}\right\} \\
&<\frac{1}{\varepsilon_{1}}\left\{\mu_{1} /\left(2 m_{0}-1\right) M_{m_{0}}+\mu_{2} / \hat{\lambda}_{m_{0}}^{\alpha}\right\}<\mu_{3} / \hat{\lambda}_{m_{0}}^{\alpha} .
\end{aligned}
$$

Thus outside an exceptional set of measure at most $\mu_{3} \sum_{k-m_{0}+1 \geq k_{0}} 1 / \lambda_{m_{0}}^{\alpha}$ we shall have

$$
\begin{aligned}
\sup _{k-m_{0}+1 \geq k_{0}} \frac{1}{k-m_{0}+1} \mid & \sum_{m=m_{0}}^{k}\left\{\xi_{m}-E\left(\eta_{m}\right)\right\} \mid \\
& \leqq \sup _{k-m_{0}+1=k_{0}} \frac{1}{k-m_{0}+1}\left|\sum_{m=m_{0}}^{k}\left\{\eta_{m}-E\left(\eta_{m}\right)\right\}\right|+\varepsilon_{1} .
\end{aligned}
$$


Now, by using the strong law of large numbers

$$
\begin{aligned}
\operatorname{Pr}\left\{\sup _{k-m_{0}+1 \geqq k_{0}}\right. & \left.\frac{1}{k-m_{0}+1}\left|\sum_{m=m_{0}}^{k}\left\{\xi_{m}-E\left(\eta_{m}\right)\right\}\right| \geqq \varepsilon\right\} \\
& \leqq \operatorname{Pr}\left\{\sup _{k-m_{0}+1 \geqq k_{0}} \frac{1}{k-m_{0}+1}\left|\sum_{m=m_{0}}^{k}\left\{\eta_{m}-E\left(\eta_{m}\right)\right\}\right| \geqq \varepsilon-\varepsilon_{1}\right\} \\
& \leqq \frac{D}{\left(\varepsilon-\varepsilon_{1}\right)^{2} k_{0}} .
\end{aligned}
$$

Hence outside a set $G_{k_{0}}$ where

we have

$$
m\left(G_{k_{0}}\right) \leqq \frac{\mu_{4}}{k_{0}}+\mu_{3} \sum_{k-m_{0}+1 \geqq k_{0}} \frac{1}{\lambda_{m_{0}}^{\alpha}}
$$

$$
\sup _{k-m_{0}+1 \geqq k_{0}} \frac{1}{k-m_{0}+1}\left|\sum_{m=m_{0}}^{k}\left\{\xi_{m}-E\left(\eta_{m}\right)\right\}\right|<\varepsilon .
$$

Therefore

$$
\frac{1}{k-m_{0}+1} \sum_{m=m_{0}}^{k} \xi_{m}>\frac{1}{k-m_{0}+1} \sum_{m=m_{0}}^{k} E\left(\eta_{m}\right)-\varepsilon
$$

for all $k$ such that $k-m_{0}+1 \geqq k_{0}$.

Since $E\left(\eta_{m}\right)=\delta$, we have

$$
N_{n}>\sum_{m=m_{0}}^{k} \xi_{m}>\left(k-m_{0}+1\right)(\delta-\varepsilon)=\left(k-\left[{ }_{2}^{1} k\right]\right)>(\mu \log n) /(\log \log n)
$$

for all $k$ such that $k-m_{0}+1 \geqq k_{0}$.

If $k$ is even, the statements $k-m_{0}+1 \geqq k_{0}$ and $k \geqq 2 k_{0}$ are equivalent, and if $k$ is odd, the statements $k-m_{0}+1 \geqq k_{0}$ and $k \geqq 2 k_{0}-1$ are equivalent. So $N_{n}>(\mu \log n) /(\log \log n)$ for all $k \geqq 2 k_{0}$.

If $n=n_{0}$ corresponds to $k=2 k_{0}$, then all $n>n_{0}$ will correspond to $k>2 k_{0}$. Therefore, for all $n>n_{0}$,

Since $\alpha>1$,

$$
N_{n}>(\mu \log n) /(\log \log n) \text {. }
$$

$$
\begin{aligned}
m\left(G_{k_{0}}\right) & \leqq \frac{\mu_{4}}{k_{0}}+\mu_{3} \sum_{k \geqq 2 k_{0}-1} \frac{1}{\hat{\lambda}_{m_{0}}^{\alpha}} \\
& =\frac{\mu_{4}}{k_{0}}+\mu_{3}\left\{\frac{1}{\hat{\lambda}_{k_{0}}^{\alpha}}+2\left(\frac{1}{\lambda_{k_{0}+1}^{\alpha}}+\frac{1}{\lambda_{k_{0}+2}^{\alpha}}+\frac{1}{\hat{\lambda}_{k_{0}+3}^{\alpha}}+\cdots\right)\right\} \\
& <\frac{\mu_{4}}{k_{0}}+2 \mu_{3} \sum_{k \geqq k_{0}} \frac{1}{\lambda_{k}^{\alpha}}
\end{aligned}
$$




$$
\begin{aligned}
& =\frac{\mu_{4}}{k_{0}}+2 \mu_{3} \sum_{k \geqslant k_{0}} \frac{1}{k(\log k)^{\alpha}} \\
& <\mu_{5}\left(\log \log n_{0} / \log n_{0}\right)+\mu_{6}\left(1 /\left(\log k_{0}\right)^{\alpha-1}\right) \\
& \left.\left.<\mu_{5} \frac{1}{\log \log n_{0}}+\mu_{7} \frac{1}{\log n_{0}} \frac{\left\{\log \left(\left(\mu_{1}^{\prime} \log n_{0}\right) /\left(\log \log n_{0}\right)\right)\right\}^{\alpha-1}}{\log n_{0}}\right)\right\}^{\alpha-1} . \\
& <\mu^{\prime} /\left\{\operatorname { l o g } \left(\frac{\log \log n_{0}}{\log } .\right.\right.
\end{aligned}
$$

\section{REFERENCES}

1. E. A. Evans, On the number of real roots of a random algebraic equation, Proc. London Math. Soc. (3) 15 (1965), 731-749. MR 31 \#5227.

2. B. V. Gnedenko and A. N. Kolmogorov, Limit distributions for sums of independent random variables, GITTL, Moscow, 1954; English transl., Addison-Wesley, Reading, Mass., 1954. MR 12, 839; MR 16, 52.

3. J. E. Littlewood and A. C. Offord, On the number of real roots of a random algebraic equation. II, Proc. Cambridge Philos. Soc. 35 (1939), 133-148.

4. G. Samal, On the number of real roots of a random algebraic equation, Proc. Cambridge Philos. Soc. 58 (1962), 433-442. MR 25 \#2657.

5. G. Samal and M. N. Mishra, On the lower bound of the number of real roots of a random algebraic equation with infinite variance, Proc. Amer. Math. Soc. 33 (1972), 523-528.

Department of Mathematics, Ravenshaw College, Cuttack 3, Orissa, India

Bureau of Statistics, Bhubaneswar, India 